\title{
CARACTERÍSTICAS FÍSICAS E CARBONO ORGÂNICO DE SOLOS SOB DIFERENTES TIPOS DE USO DA TERRA ${ }^{(1)}$
}

\author{
Jacson Tavares de Oliveira ${ }^{(2)}$, Ana Maria Souza dos Santos Moreau ${ }^{(3)}$, Arlicélio de \\ Queiroz Paiva $^{(3)}$, Agna Almeida Menezes ${ }^{(3)}$ \& Oldair Vinhas Costa $^{(4)}$
}

\begin{abstract}
RESUMO
Na Bacia Hidrográfica Água Fria, em Barra do Choça (BA), o desenvolvimento da lavoura cafeeira e da pastagem tem sido feito mediante a supressão da vegetação nativa, inclusive em áreas de proteção ambiental. O objetivo deste trabalho foi avaliar os atributos físicos: granulometria, argila dispersa em água, grau de floculação, diâmetro médio ponderado e índice de estabilidade de agregados e $\mathrm{C}$ orgânico dos solos submetidos a diferentes tipos de uso da terra na referida bacia. Para as amostragens, foram selecionadas seis glebas: duas de café (cultivo mecanizado e não mecanizado), duas de pastagens e duas de matas nativas, empregadas como referência. Constatou-se que o uso da terra diminuiu a estabilidade de agregados, evidenciada pela redução no diâmetro médio ponderado, pelo aumento dos agregados de menor tamanho e pelos menores teores de C orgânico na seqüência mata-pastagem-cafezal.
\end{abstract}

Termos de indexação: agregação do solo, café, plantio convencional.

(1) Parte da Dissertação de Mestrado, do primeiro autor, em Desenvolvimento Regional e Meio Ambiente junto à Universidade Estadual de Santa Cruz - UESC. Trabalho apresentado no XXXI Congresso Brasileiro de Ciência do Solo, 2007 (Gramado, RS).

(2) Professor do CEFET. Avenida Amazonas, 3150, Ibirapuera. CEP:45030-220, Vitória da Conquista (BA). E-mail: joliveira@cefetba.br

(3) Professor do Departamento de Ciências Agrárias e Ambientais da UESC. E-mail: amoreau@uesc.br; arli@uesc.br; agna@uesc.br

(4) Professor do CCAAB da Universidade Federal do Recôncavo da Bahia. Campus Universitário. CEP: 44380-000 Cruz das Almas (BA). E-mail: oldair@ufrb.edu.br 


\title{
SUMMARY: SOIL PHYSICAL CHARACTERISTICS AND ORGANIC CARBON CONTENT UNDER DIFFERENT LAND USES
}

\begin{abstract}
In the watershed Água Fria, in Barra do Choça (BA), native vegetation and environmentally protected areas have been replaced by coffee and pasture cultivation. This study evaluated the physical characteristics, particle size distribution, water dispersible clay, flocculation, mean weight diameter and aggregate stability index and organic $C$ concentration of soils under different land uses, in the area surroundings the above watershed. Soil samples were collected from six different fields: two coffee plantations (manual and mechanical cultivation), two pastures and two native forest areas were used as references. The different land uses reduced the aggregate stability, as indicated by the reduction of the mean weight diameter, increase of small-sized aggregates and lower organic C contents, in the order forest $>$ pasture $>$ coffee plantation.
\end{abstract}

Index terms: soil aggregation, coffee plant, conventional tillage.

\section{INTRODUÇÃO}

A incorporação de espaços naturais para os cultivos agrícolas e o estabelecimento de pastagens para o gado alteram as características físicas e químicas dos solos, muitas vezes resultando em degradação que se manifesta pela perda de fertilidade, compactação e fracionamento dos agregados, comprometendo a infiltração de água e o crescimento adequado do sistema radicular dos vegetais (Bertol et al., 2001; Bronick \& Lal, 2005).

Nos preparos convencionais, é comum a retirada da vegetação nativa para estabelecimento de atividades que deixam o solo exposto ao impacto direto das chuvas, trazendo como conseqüência o rompimento dos agregados (Wohlenberg et al., 2004). Aliado a isso, o constante revolvimento da terra contribui para a redução do teor de matéria orgânica, reconhecida como um dos principais agentes de formação e estabilização de agregados (Roth et al., 1991; Grieve et al., 2005).

De acordo com Bronick \& Lal (2005), a agregação resulta do rearranjo de partículas, floculação e cimentação mediado pelo $\mathrm{C}$ orgânico do solo, biota, ponte iônica, argila e carbonatos, sendo o $\mathrm{C}$, ao mesmo tempo, agente de ligação e núcleo na formação dos agregados.

Em locais de produção de água para abastecimento, como a Bacia Água Fria, em Barra do Choça-BA, o estudo das características físicas dos solos é fundamental. De acordo com Bronick \& Lal (2005), alterações no solo causadas por manejo inadequado podem comprometer a quantidade e a qualidade da água, já que a estrutura e a textura do solo têm influência no fluxo de água no solo, na sua disponibilidade e no seu armazenamento.

Assim, objetivou-se no presente trabalho verificar as alterações nas propriedades físicas e no C orgânico de Latossolos Amarelos sob diferentes sistemas de uso e manejo.

\section{MATERIAL E MÉTODOS}

O estudo foi realizado nas Sub-Bacias Hidrográficas do Rio dos Monos (SBHRM) e do Rio Água Fria (SBHRAF) que formam a Bacia Agua Fria, em Barra do Choça, Bahia (Figura 1), situada nas unidades geomorfológicas Planaltos dos Geraizinhos e Piemonte Oriental do Planalto de Vitória da Conquista. Na área, predominam os Latossolos Amarelos alumínicos e distróficos, desenvolvidos a partir das Coberturas Detríticas tércio-quaternárias sobre terrenos do Complexo Caraíba-Paramirim, do Pré-Cambriano Inferior (Brasil, 1981). O Clima, segundo Köppen, é o Aw, com concentração de chuvas no período de novembro a abril, enquanto os meses seguintes aparecem como os mais secos, todos com valores inferiores a $60 \mathrm{~mm}$.

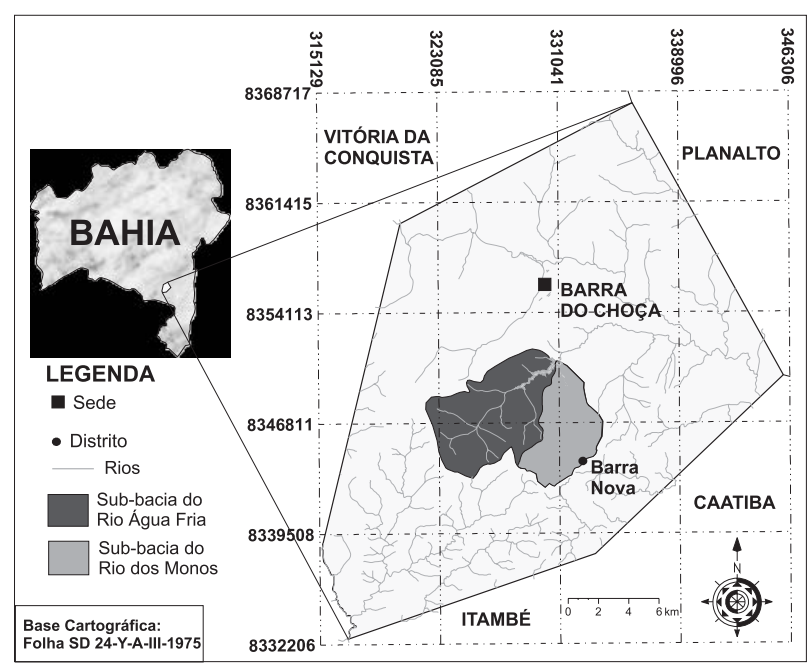

Figura 1. Mapa de Localização da Bacia Água Fria em Barra do Choça-Bahia. 
Os atributos físicos e C orgânico dos solos submetidos a diferentes tipos de uso foram avaliados em seis áreas amostrais de Latossolos Amarelos, selecionadas em topo de elevação, em altitude em torno de $900 \mathrm{~m}$. Os tipos de uso podem ser assim caracterizados: (a) mata nativa na SBHRM e na SBHRAF: área sob fragmentos da vegetação original preservada (Floresta Estacional Decidual), que permaneceu como Reserva Legal, ao processo de expansão da agricultura na região - esta área foi escolhida como referência para comparação das alterações dos atributos estudados; (b) pastagem de capim-gordura (Melinis minutiflora) na SBHRM e de braquiária (Brachiaria decumbens) na SBHRAF: áreas com oito anos de uso, manejadas com bovinos, sem controle de lotação. Tais áreas foram implantadas no sistema convencional para a atividade pecuária, onde se suprimiu a vegetação original e, após a queima, foram plantadas gramíneas. Nesse sistema, não há correção da acidez do solo nem adubação; (c) café em cultivo não mecanizado, SBHRAF: caracterizado pelo espaçamento $2,5 \times 1,0 \mathrm{~m}$ (semi-adensado), com densidade populacional de 4.000 plantas por hectare. O cafezal foi instaldao há cinco anos e, quando da sua instalação, aplicou-se o equivalente $3.400 \mathrm{~kg} \mathrm{ha}^{-1} \mathrm{de}$ calcário dolomítico. Nas covas de plantio, foram aplicados $10 \mathrm{dm}^{3}$ de esterco bovino, $300 \mathrm{~g}$ de calcário dolomítico, $200 \mathrm{~g}$ de superfosfato simples, $40 \mathrm{~g}$ de cloreto de potássio, além dos micronutrientes $\mathrm{Zn}$ e B. As adubações de manutenção foram realizadas de acordo com as recomendações preconizadas para o cafeeiro. $\mathrm{O}$ controle de plantas invasoras é feito duas vezes ao ano com aplicação de herbicida. Não há trânsito de máquinas agrícolas e o café é irrigado. (d) café em cultivo mecanizado - SBHRM: caracterizado pelo espaçamento 4,0 x 1,5 m, com densidade de 1.666 covas por hectare. $\mathrm{O}$ cafezal tem cinco anos e adotouse o mesmo procedimento descrito acima, para o café semi-adensado, quando de sua instalação. Foram utilizados tratores para aração e gradagem para o plantio da lavoura e uso de maquinários pesados nas entrelinhas para manutenção do cafezal (tratos culturais e colheita). A adubação de manutenção é feita sem periodicidade.

As amostras de solo coletadas foram analisadas para determinação da granulometria, argila dispersa em água, grau de floculação, distribuição de classes de tamanhos de agregados e C orgânico do solo, segundo método preconizado por Embrapa (1997). O cálculo do diâmetro médio ponderado dos agregados do solo (DMP) foi feito pela fórmula proposta por Youker \& McGuinness (1957).

No quadro 1, encontram-se as médias dos atributos químicos obtidos, por horizonte, dos Latossolos Amarelos distróficos estudados. Tais valores foram utilizados na análise estatística de correlação de Pearson.

\section{RESULTADOS E DISCUSSÃO}

\section{Atributos físicos}

Os Latossolos Amarelos amostrados apresentaram teor de argila superior a $350 \mathrm{~g} \mathrm{~kg}^{-1}$ em todos os horizontes (Quadro 2), portanto, com classe textural argilosa, exceto o horizonte $\mathrm{Ap}_{1}$ do LAd5 que apresentou textura média.

A relação silte/argila apresentou valores baixos em todos os horizontes, uma vez que esses solos são produtos da alteração de sedimentos pré-intemperizados e edafisados, portanto, pobres em minerais primários, além de serem velhos e bastante intemperizados.

Com relação ao grau de floculação da argila (GF), os horizontes superficiais dos solos apresentaram menor valor, quando comparados aos horizontes subsuperficiais diagnósticos.

Quadro 1. Médias dos teores dos atributos químicos, por horizontes, dos solos das sub-bacias do Rio dos Monos e do Rio Água Fria, em Barra do Choça-Bahia

\begin{tabular}{|c|c|c|c|c|c|c|c|c|c|c|c|c|c|c|c|c|}
\hline \multirow[t]{2}{*}{ Horiz. } & \multicolumn{2}{|c|}{ pH $(1: 2,5)$} & \multirow[t]{2}{*}{$\Delta \mathbf{p H}$} & \multirow[t]{2}{*}{ P disp. } & \multirow[t]{2}{*}{ C org. } & \multicolumn{11}{|c|}{ Complexo Sortivo } \\
\hline & $\mathrm{H}_{2} \mathrm{O}$ & $\mathrm{KCl}$ & & & & $\mathbf{C a}^{2+}$ & $\mathrm{Mg}^{2+}$ & $\mathbf{K}^{+}$ & $\mathrm{Na}^{+}$ & $\mathrm{Al}^{3+}$ & $\mathbf{H}^{+}$ & SB & $\mathbf{t}$ & $\mathbf{T}$ & V & $\mathbf{m}$ \\
\hline & & & & $m g \mathrm{~kg}^{-1}$ & $\mathrm{~g} \mathrm{dm}^{-3}$ & $\longrightarrow$ & & & $-\mathrm{cmo}$ & $\mathrm{dm}^{-3}$ & & & & & - $\%$ & $\longrightarrow$ \\
\hline $\mathrm{A}$ & 5,0 & 4,3 & $-0,7$ & 6,4 & 24,5 & 2,4 & 0,7 & 0,6 & 0,4 & 1,2 & 11,3 & 4,1 & 5,3 & 15,4 & 24,7 & 32,4 \\
\hline $\mathrm{AB}$ & 5,2 & 4,3 & $-0,9$ & 0,8 & 18,2 & 1,4 & 0,4 & 0,2 & 0,2 & 1,2 & 9,6 & 2,2 & 3,4 & 11,7 & 16,7 & 45,3 \\
\hline BA & 5,1 & 4,3 & $-0,8$ & 0,2 & 10,4 & 0,3 & 0,2 & 0,1 & 0,2 & 1,1 & 6,2 & 0,8 & 1,9 & 7,0 & 11,6 & 56,7 \\
\hline $\mathrm{Bw}_{1}$ & 4,9 & 4,4 & $-0,5$ & 0,0 & 5,5 & 0,3 & 0,2 & 0,1 & 0,3 & 0,6 & 4,0 & 0,8 & 1,4 & 4,9 & 17,3 & 48,2 \\
\hline $\mathrm{Bw}_{2}$ & 4,9 & 4,6 & $-0,4$ & 0,8 & 2,9 & 0,3 & 0,2 & 0,0 & 0,2 & 0,3 & 2,9 & 0,8 & 1,1 & 3,6 & 21,2 & 33,7 \\
\hline $\mathrm{BC}$ & 5,1 & 4,6 & $-0,5$ & 0,5 & 3,0 & 0,1 & 0,1 & 0,0 & 0,2 & 0,3 & 2,4 & 0,5 & 0,8 & 2,9 & 14,6 & 42,8 \\
\hline
\end{tabular}

SB = Soma de bases; $\mathrm{t}=$ CTC efetiva; $\mathrm{T}=\mathrm{CTC}$ a pH 7,0; $\mathrm{V}=$ Saturação por bases; $\mathrm{m}=$ saturação por $\mathrm{Al}$. 
Quadro 2. Características físicas e carbono orgânico dos solos das sub-bacias do Rio dos Monos (LAd1, LAd2 e LAd3) e do Rio Água Fria (LAd4, LAd5, LAd6), em Barra do Choça-Bahia

\begin{tabular}{|c|c|c|c|c|c|c|c|c|c|}
\hline HORIZONTE & AG & $\mathbf{A F}$ & AT & $\mathbf{S}$ & ARG & ADA & S/A & GF & C org. \\
\hline & & & - g & & & & & $\%$ & $\mathrm{~g} \mathrm{dm}^{-3}$ \\
\hline \multicolumn{10}{|c|}{ LAd1 - Latossolo Amarelo distrófico argissólico - pastagem capim-gordura } \\
\hline$A(0-15 \mathrm{~cm})$ & 414 & 113 & 527 & 120 & 353 & 115 & 0,34 & 67 & 22,08 \\
\hline $\mathrm{AB}(15-24 \mathrm{~cm})$ & 365 & 128 & 493 & 93 & 414 & 275 & 0,22 & 34 & 15,72 \\
\hline BA $(24-45 \mathrm{~cm})$ & 325 & 129 & 454 & 92 & 454 & 296 & 0,20 & 35 & 10,92 \\
\hline $\mathrm{Bw}_{1}(45-100 \mathrm{~cm})$ & 282 & 109 & 391 & 29 & 580 & 7 & 0,05 & 99 & 4,68 \\
\hline $\mathrm{Bw}_{2}(100-150 \mathrm{~cm})$ & 284 & 103 & 387 & 37 & 576 & 13 & 0,06 & 98 & 3,48 \\
\hline $\mathrm{BC}(150-180 \mathrm{~cm}+)$ & 260 & 98 & 358 & 19 & 623 & 138 & 0,03 & 78 & 3,00 \\
\hline \multicolumn{10}{|c|}{ LAd2 - Latossolo Amarelo distrófico típico - mata nativa } \\
\hline $\mathrm{A}(0-12 \mathrm{~cm})$ & 345 & 105 & 450 & 115 & 435 & 214 & 0,26 & 51 & 28,80 \\
\hline $\mathrm{AB}(12-25 \mathrm{~cm})$ & 284 & 109 & 393 & 95 & 512 & 367 & 0,19 & 28 & 15,96 \\
\hline $\mathrm{BA}(25-41 \mathrm{~cm})$ & 283 & 101 & 384 & 97 & 519 & 244 & 0,19 & 53 & 12,60 \\
\hline $\mathrm{Bw}_{1}(41-66 \mathrm{~cm})$ & 224 & 106 & 330 & 67 & 603 & 458 & 0,11 & 24 & 9,12 \\
\hline $\mathrm{Bw}_{2}(66-110 \mathrm{~cm})$ & 219 & 105 & 324 & 68 & 608 & 0 & 0,11 & 100 & 6,72 \\
\hline BC $(110-144 \mathrm{~cm})$ & 184 & 87 & 271 & 68 & 661 & 240 & 0,10 & 64 & 3,00 \\
\hline $\mathrm{C}(144-166 \mathrm{~cm})$ & 194 & 83 & 277 & 90 & 633 & 24 & 0,14 & 96 & 1,68 \\
\hline \multicolumn{10}{|c|}{ LAd3 - Latossolo Amarelo distrófico típico - café (cultivo mecanizado) } \\
\hline Ap $(0-18 \mathrm{~cm})$ & 359 & 104 & 463 & 90 & 447 & 224 & 0,20 & 50 & 18,60 \\
\hline $\mathrm{BA}(18-39 \mathrm{~cm})$ & 282 & 99 & 381 & 70 & 549 & 139 & 0,13 & 75 & 12,12 \\
\hline $\mathrm{Bw}_{1}(39-130 \mathrm{~cm})$ & 236 & 99 & 335 & 29 & 636 & 0 & 0,05 & 100 & 3,48 \\
\hline $\mathrm{Bw}_{2}(130-200 \mathrm{~cm}+)$ & 212 & 80 & 292 & 38 & 670 & 3 & 0,06 & 100 & 1,08 \\
\hline \multicolumn{10}{|c|}{ LAd4 - Latossolo Amarelo distrófico húmico - pastagem braquiária } \\
\hline $\mathrm{A}(0-37 \mathrm{~cm})$ & 335 & 145 & 480 & 67 & 453 & 109 & 0,14 & 76 & 18,60 \\
\hline $\mathrm{AB}(37-66 \mathrm{~cm})$ & 347 & 137 & 484 & 86 & 430 & 253 & 0,20 & 41 & 25,80 \\
\hline $\mathrm{AB}(66-130 \mathrm{~cm})$ & 242 & 166 & 408 & 30 & 562 & 310 & 0,05 & 45 & 15,60 \\
\hline $\mathrm{BA}(130-157 \mathrm{~cm})$ & 209 & 156 & 365 & 12 & 623 & 290 & 0,02 & 53 & 5,64 \\
\hline $\mathrm{Bw}(157-212 \mathrm{~cm}+)$ & 222 & 157 & 379 & 6 & 615 & 0 & 0,01 & 100 & 2,88 \\
\hline \multicolumn{10}{|c|}{ LAd5 - Latossolo Amarelo distrófico típico - café (cultivo não mecanizado) } \\
\hline Ap1 $(0-15 \mathrm{~cm})$ & 385 & 128 & 513 & 138 & 349 & 82 & 0,39 & 77 & 48,60 \\
\hline Ap2 $(15-34 \mathrm{~cm})$ & 319 & 147 & 466 & 73 & 461 & 157 & 0,16 & 66 & 24,00 \\
\hline $\mathrm{AB}(34-55 \mathrm{~cm})$ & 287 & 144 & 431 & 67 & 502 & 176 & 0,13 & 65 & 18,72 \\
\hline $\mathrm{BA}(55-95 \mathrm{~cm})$ & 260 & 142 & 402 & 31 & 567 & 27 & 0,06 & 95 & 6,24 \\
\hline $\mathrm{Bw}_{1}(95-121 \mathrm{~cm})$ & 246 & 137 & 383 & 206 & 553 & 0 & 0,37 & 100 & 2,76 \\
\hline $\mathrm{Bw}_{2}(121-180 \mathrm{~cm}+)$ & 220 & 113 & 333 & 273 & 593 & 0 & 0,46 & 100 & 1,44 \\
\hline \multicolumn{10}{|c|}{ LAd6 - Latossolo Amarelo distrófico típico - mata nativa } \\
\hline A $(0-26 \mathrm{~cm})$ & 347 & 110 & 457 & 93 & 450 & 82 & 0,21 & 95 & 28,80 \\
\hline $\mathrm{AB}(26-46 \mathrm{~cm})$ & 321 & 121 & 442 & 95 & 463 & 175 & 0,20 & 96 & 17,40 \\
\hline $\mathrm{BA}(46-74 \mathrm{~cm})$ & 304 & 123 & 427 & 92 & 481 & 252 & 0,19 & 94 & 14,64 \\
\hline $\mathrm{Bw}_{1}(74-118 \mathrm{~cm})$ & 333 & 122 & 455 & 83 & 462 & 209 & 0,18 & 86 & 7,56 \\
\hline $\mathrm{Bw}_{2}(118-195 \mathrm{~cm}+)$ & 257 & 115 & 372 & 183 & 445 & 0 & 0,41 & 97 & 1,68 \\
\hline
\end{tabular}

$\mathrm{AG}=$ areia grossa; $\mathrm{AF}=$ areia fina; $\mathrm{AT}=$ areia total; $\mathrm{S}=$ silte; $\mathrm{ARG}=$ argila $\mathrm{ADA}=$ argila dispersa em água; $\mathrm{S} / \mathrm{A}=$ relação silte/ argila; $\mathrm{GF}=$ grau de floculação; $\mathrm{C}$ Orgânico = carbono orgânico. 
De modo geral, as características que melhor se correlacionaram com o grau de floculação foram o teor de carbono e a CTC (T) (Quadro 3). No entanto, o efeito do $\mathrm{C}$ no GF diferiu por posição de horizonte; enquanto, nos superficiais, a correlação foi positiva, nos subsuperficiais, foi negativa (Figura 2).

Em média, os horizontes superficiais são menos floculados $(\mathrm{GF}=51,5 \%)$ que os subsuperficiais diagnósticos ( $\mathrm{GF}=84 \%)$. De modo geral, a floculação é maior quando há menor densidade de cargas negativas e, conseqüentemente, menor repulsão mútua entre as partículas com mesmo campo elétrico, maior concentração de eletrólitos, que comprime a dupla camada difusa, menores valores de $\mathrm{pH}$, o que diminui o número de cargas negativas no meio e menor concentração de íons dispersantes como o $\mathrm{Na}^{+}$.

Dessa forma, os horizontes subsuperficiais são mais floculados por terem, em média, menor densidade de cargas negativas $\left(\mathrm{T}=4,1 \mathrm{cmol}_{\mathrm{C}} \mathrm{dm}^{-3}\right)$, menor $\mathrm{pH}(4,9) \mathrm{e}$ menor teor de $\mathrm{Na}$ trocável $\left(0,2 \mathrm{cmol}_{\mathrm{c}} \mathrm{dm}^{-3}\right)$, quando comparado aos horizontes de superfície (Quadro 1). Por outro lado, os horizontes subsuperficiais possuem menor concentração de eletrólitos $\left(t=1,2 \mathrm{cmol}_{\mathrm{c}}\right.$ $\left.\mathrm{dm}^{-3}\right)$, maior saturação por sódio (7,0 \%) e menor teor de $\mathrm{Al}^{3+}\left(0,5 \mathrm{cmol}_{\mathrm{c}} \mathrm{dm}^{-3}\right)$ que os horizontes superficiais, características antagônicas à floculação.

Por isso, acredita-se que o aumento no teor de C orgânico, nestes horizontes (subsuperficiais), seja responsável pelo aumento no número de cargas negativas e conseqüente maior dispersão das argilas, visto que o aumento de $1 \mathrm{dag} / \mathrm{kg}$ de C eleva a CTC destes horizontes em 5,02 $\mathrm{cmol}_{\mathrm{c}} \mathrm{dm}^{-3}$ (Figura 3), o que significa um aumento médio de $123 \%$ na CTC. Nos horizontes superficiais, a contribuição do C na CTC é aproxima-damente igual, $4,77 \mathrm{cmol}_{c} \mathrm{dm}^{-3}$ (Figura 3); no entanto, significa um incremento relativo de $35 \%$ na CTC. Assim, o efeito do C nestes horizontes é oposto, ou seja, o $\mathrm{C}$ atua floculando; dessa forma, acredita-se que a magnitude no aumento da CTC dos horizontes subsuperficiais leve à dispersão do solo, apesar de serem mais floculados que os horizontes superficiais.

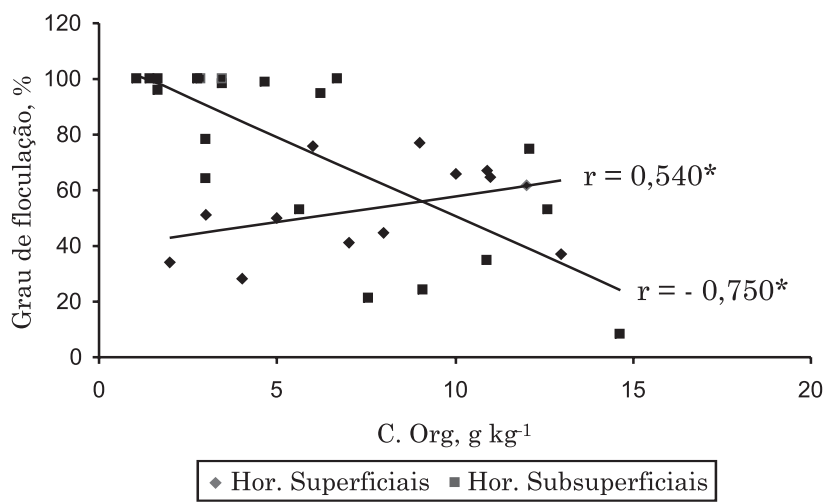

Figura 2. Relação entre grau de floculação e carbono orgânico dos solos das sub-bacias do Rio dos Monos e do Rio Água Fria, em Barra do Choça-Bahia.

Quadro 3. Coeficientes de correlação de Pearson entre atributos do solo e grau de floculação (GF), diâmetro médio ponderado de agregados (DMP) e índice de estabilidade de agregados (IEA)

\begin{tabular}{|c|c|c|c|c|c|c|c|c|c|}
\hline \multirow{2}{*}{ Atributo } & \multicolumn{3}{|c|}{ GF (\%) } & \multicolumn{3}{|c|}{ DMP (mm) } & \multicolumn{3}{|c|}{ IEA $(\%)$} \\
\hline & Todos & Hor.Super. & Hor. Sub & Todos & Hor.Super. & Hor. Sub & Todos & Hor.Super. & Hor. Sub \\
\hline $\mathrm{N}$ & 33 & 13 & 20 & 33 & 13 & 20 & 33 & 13 & 20 \\
\hline Areia Total, g/kg & $-0,39$ & 0,49 & $-0,40$ & 0,48 & 0,37 & $-0,05$ & $-0,01$ & 0,07 & $-0,42$ \\
\hline Areia Grossa, g/kg & $-0,30$ & 0,34 & $-0,28$ & 0,59 & 0,43 & 0,22 & 0,16 & 0,20 & $-0,15$ \\
\hline Areia Fina, $g / k g$ & $-0,33$ & 0,15 & $-0,35$ & $-0,11$ & $-0,32$ & $-0,40$ & $-0,41$ & $-0,38$ & $-0,56$ \\
\hline Silte, $\mathrm{g} / \mathrm{kg}$ & $-0,31$ & $-0,03$ & $-0,39$ & $-0,13$ & $-0,03$ & $-0,47$ & $-0,26$ & 0,17 & $-0,52$ \\
\hline Argila, $\mathrm{g} / \mathrm{kg}$ & 0,55 & $-0,20$ & 0,64 & $-0,22$ & $-0,14$ & 0,36 & 0,18 & $-0,21$ & 0,61 \\
\hline Silte+Argila, $\mathrm{g} / \mathrm{kg}$ & $-0,45$ & 0,20 & $-0,48$ & 0,15 & 0,14 & $-0,41$ & $-0,21$ & 0,21 & $-0,59$ \\
\hline Silte/argila & $-0,38$ & 0,01 & $-0,57$ & $-0,07$ & $-0,03$ & $-0,50$ & $-0,23$ & 0,18 & $-0,61$ \\
\hline $\mathrm{pH}$ sal & 0,34 & $-0,09$ & 0,45 & $-0,44$ & $-0,51$ & $-0,30$ & $-0,19$ & $-0,44$ & 0,07 \\
\hline$\Delta \mathrm{pH}$ & 0,35 & $-0,02$ & 0,46 & $-0,29$ & $-0,29$ & $-0,12$ & 0,14 & 0,19 & 0,22 \\
\hline C. Org, g dm-3 & $-0,39$ & 0,54 & $-0,75$ & 0,49 & 0,00 & 0,28 & 0,18 & $-0,11$ & 0,03 \\
\hline $\mathrm{Al}^{3+}, \mathrm{cmol}_{\mathrm{c}} \mathrm{dm}^{-3}$ & $-0,41$ & $-0,16$ & $-0,48$ & 0,36 & 0,13 & 0,35 & 0,22 & 0,27 & 0,07 \\
\hline $\mathrm{H}+\mathrm{Al}$ a pH $7,0, \mathrm{cmol}_{\mathrm{c}} \mathrm{dm}^{-3}$ & $-0,52$ & 0,02 & $-0,70$ & 0,59 & 0,48 & 0,04 & 0,22 & 0,42 & $-0,21$ \\
\hline $\mathrm{T}, \mathrm{cmol}_{\mathrm{c}} \mathrm{dm}^{-3}$ & $-0,34$ & 0,56 & $-0,61$ & 0,47 & 0,04 & 0,00 & 0,14 & $-0,08$ & $-0,25$ \\
\hline $\mathrm{m}, \%$ & $-0,35$ & $-0,36$ & $-0,64$ & $-0,13$ & $-0,13$ & 0,22 & $-0,05$ & 0,09 & $-0,04$ \\
\hline $\mathrm{Na}, \mathrm{cmol}_{\mathrm{c}} \mathrm{dm}^{-3}$ & 0,14 & $-0,48$ & 0,32 & $-0,04$ & $-0,33$ & $-0,11$ & $-0,09$ & $-0,35$ & $-0,05$ \\
\hline
\end{tabular}

Valores em negrito significativos a $90 \%$. 


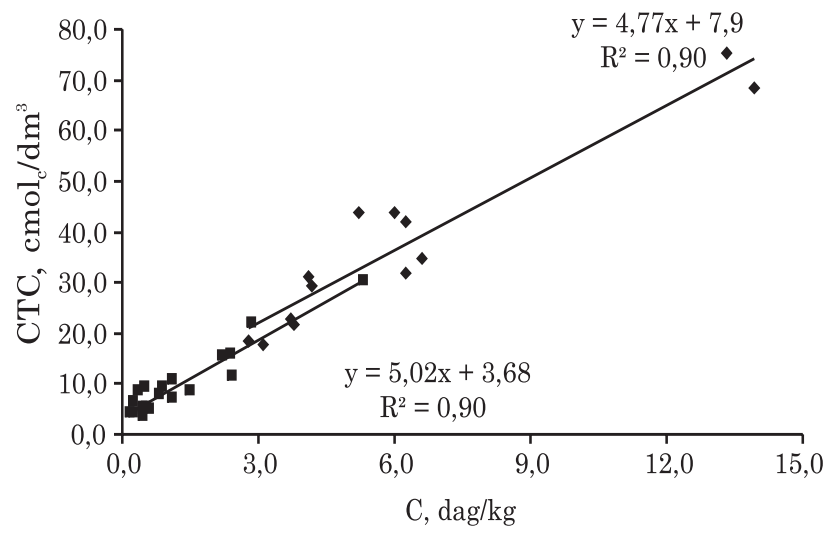

- Hor. Superficiais $\quad$ Hor. Subsuperficiais

Figura 3. Relação entre teor de carbono e CTC dos solos das sub-bacias do Rio dos Monos e do Rio Água Fria, em Barra do Choça-Bahia.

Os resultados obtidos com a análise de agregados (Quadro 4 ) mostram que a distribuição de classes de tamanhos de agregados, o diâmetro médio ponderado (DMP) e o índice de estabilidade de agregados (IEA) variaram com os diferentes tipos de uso do solo.

Na SBHRM (Quadro 4), o solo sob mata nativa (LAd2) apresentou o maior valor do IEA, atingindo valores superiores a $79 \%$ (horizonte A) e $84 \%$ (horizonte AB) e DMP de 1,26 mm no horizonte A e $0,93 \mathrm{~mm}$ no $\mathrm{AB}$. O fato de o horizonte $\mathrm{AB}$ ter apresentado maior índice de estabilidade de agregados em relação ao horizonte A pode ser devido ao seu maior teor de argila. Além disso, estes horizontes também mostraram maior percentual de agregados de maior diâmetro (4,76-2,00 e 2,00$1,00 \mathrm{~mm}$ ). As razões para estes níveis de agregação podem estar relacionadas com os maiores teores de C orgânico encontrados nestes horizontes (Quadro 2), uma vez que a matéria orgânica é agente primordial na formação e estabilização de agregados, e, na mata, o aporte constante de matéria orgânica concorre para a melhor agregação dos solos.

Quanto aos horizontes inferiores, houve tendência de aumento do percentual de agregados de menor diâmetro (0,50-0,25 e <0,25 mm) e diminuição do IEA e do DMP com a profundidade (Quadro 4). A explicação está no comportamento do C orgânico e da argila no perfil do solo: enquanto o teor de argila aumenta com a profundidade, o de C orgânico diminui (Quadro 2), contribuindo, dessa forma, para a formação de agregados de menor tamanho, já que a cimentação nos horizontes inferiores é verificada prioritariamente pela ação agregadora da argila. Os altos valores do grau de floculação da argila encontrados nestes horizontes também fortalecem essa hipótese. Além disso, a menor agregação neste caso pode estar relacionada com o menor crescimento das raízes das gramíneas em profundidade.

Tomando os valores do solo sob mata (LAd2) como referência, a pastagem (LAd1) exibiu valores menores de IEA, que ficou em torno de $77 \%$ no horizonte A, mas, por outro lado, esse mesmo horizonte teve $25,3 \%$ de agregados na faixa de 4,76 $2,00 \mathrm{~mm}$ contra $17,7 \%$ da mata. No AB do LAd1, a agregação também foi maior na classe 2,00-1,00 $\mathrm{mm}$. Este efeito cimentante pode estar associado ao poder que as gramíneas têm de agregar as partículas minerais e a fração orgânica nos horizontes superficiais e subsuperficiais (Silva \& Mielniczuk, 1997; Cruz et al., 2003; Wohlenberg et al., 2004). Além disso, os teores de $\mathrm{C}$ orgânico não diminuíram tanto em relação ao LAd2, contribuindo também na agregação. Estes fatores também devem ter concorrido para aumentar o DMP no horizonte A da pastagem. Já os horizontes inferiores apresentaram a mesma tendência de aumento da floculação da argila, diminuição do IEA e do DMP com a profundidade e aumento do percentual de agregados de menor diâmetro.

O efeito mais visível na alteração das propriedades físicas do solo foi verificado no solo sob café (LAd3). Os valores do IEA que estavam em torno de $80 \%$ na mata nativa aqui foram reduzidos para valores inferiores a $67 \%$, com reflexos na redução do DMP, que caiu pela metade no horizonte Ap e cerca de um terço no BA. O tamanho dos agregados também foi drasticamente reduzido, confirmando os efeitos destrutivos do cultivo para os agregados de maior dimensão (John et al., 2005). Para a classe 4,76-2,00 mm, os valores do horizonte Ap representam menos de $10 \%$ do total observado no horizonte A do LAd2. Mais de $50 \%$ dos agregados do LAd3 estão nas classes de menor diâmetro (0,50-0,25 e <0,25 mm), e no BA este número sobe para mais de $60 \%$, atestando que o preparo do solo por meio de aração e gradagem para a implantação da lavoura e o uso de maquinários pesados para manutenção do cafezal concorrem para a destruição da estrutura do solo (John et al., 2005). Este revolvimento pulveriza e expõe o solo às variações de temperatura e umidade, destruindo os agregados de maior tamanho (Wendling et al., 2005). Os horizontes inferiores seguiram a mesma tendência verificada na mata e na pastagem quanto ao grau de floculação, distribuição de classes de tamanhos de agregados e DMP. A exceção foi o aumento do IEA no horizonte $\mathrm{Bw}_{2}$, que apresentou $73,79 \%$, a maior de todo o perfil. Isto pode ser justificado pelo maior teor de argila (670 $\mathrm{g} \mathrm{kg}^{-1}$ ).

Na SBHRAF (Quadro 4), o comportamento dos agregados do solo foi muito parecido com as variações verificadas na SBHRM, onde a magnitude dos efeitos causados pelos diferentes tipos de uso, na estrutura do solo, cresceu no sentido matapastagem-cafezal. No entanto, é importante destacar algumas particularidades verificadas na referida sub-bacia. 
Quadro 4. Análise de agregados dos solos das sub-bacias do Rio dos Monos (LAd1, LAd2 e LAd3) e do Rio Água Fria (LAd4, LAd5, LAd6), em Barra do Choça-Bahia

\begin{tabular}{|c|c|c|c|c|c|c|c|}
\hline \multirow{2}{*}{ Horizonte } & \multicolumn{5}{|c|}{ Distribuição de Classes de Tamanhos de Agregados (mm) } & \multirow{2}{*}{ DMP } & \multirow{2}{*}{ IEA } \\
\hline & $4,76-2,00$ & $2,00-1,00$ & $1,00-0,50$ & $0,50-0,25$ & $<0,25$ & & \\
\hline \multicolumn{8}{|c|}{ LAd1 - Latossolo Amarelo distrófico argissólico - pastagem capim-gordura } \\
\hline A $\quad(0-15 \mathrm{~cm})$ & 25,3 & 23,9 & 8,4 & 16,1 & 26,3 & 1,35 & 77,33 \\
\hline $\mathrm{AB}(15-24 \mathrm{~cm})$ & 7,0 & 36,0 & 13,7 & 23,3 & 20,0 & 0,99 & 75,97 \\
\hline BA $(24-45 \mathrm{~cm})$ & 6,0 & 32,7 & 18,8 & 16,3 & 26,3 & 0,93 & 76,08 \\
\hline $\mathrm{Bw}_{1}(45-100 \mathrm{~cm})$ & 2,5 & 15,7 & 15,4 & 28,6 & 37,8 & 0,60 & 56,02 \\
\hline $\left.\mathrm{Bw}_{2} 100-150 \mathrm{~cm}\right)$ & 4,8 & 28,3 & 10,7 & 14,6 & 41,6 & 0,77 & 74,27 \\
\hline $\mathrm{BC}(150-180 \mathrm{~cm}+)$ & 2,6 & 15,6 & 15,7 & 24,2 & 41,9 & 0,71 & 72,52 \\
\hline \multicolumn{8}{|c|}{ LAd2 - Latossolo Amarelo distrófico típico - mata nativa } \\
\hline A $\quad(0-12 \mathrm{~cm})$ & 17,7 & 26,5 & 13,3 & 17,2 & 25,3 & 1,26 & 79,41 \\
\hline $\mathrm{AB}(12-25 \mathrm{~cm})$ & 16,5 & 30,1 & 14,0 & 15,6 & 23,8 & 0,93 & 84,85 \\
\hline BA $(25-41 \mathrm{~cm})$ & 8,0 & 27,9 & 15,8 & 17,6 & 30,7 & 0,91 & 82,94 \\
\hline $\mathrm{Bw}_{1}(41-66 \mathrm{~cm})$ & 7,8 & 26,0 & 14,3 & 20,5 & 31,4 & 0,88 & 84,75 \\
\hline $\mathrm{Bw}_{2}(66-110 \mathrm{~cm})$ & 3,8 & 16,8 & 14,6 & 20,8 & 44,1 & 0,62 & 70,91 \\
\hline BC $(110-144 \mathrm{~cm})$ & 9,7 & 18,6 & 15,8 & 17,3 & 38,6 & 0,76 & 78,27 \\
\hline C $(144-166 \mathrm{~cm})$ & 5,2 & 16,2 & 15,2 & 20,3 & 43,1 & 0,63 & 68,65 \\
\hline
\end{tabular}

LAd3 - Latossolo Amarelo distrófico típico- café (cultivo mecanizado)

$$
\begin{aligned}
& \text { Ap }(0-18 \mathrm{~cm}) \\
& \text { BA }(18-39 \mathrm{~cm}) \\
& \text { Bw }_{1}(39-130 \mathrm{~cm}) \\
& \text { Bw }_{2}(130-200 \mathrm{~cm}+)
\end{aligned}
$$

$$
1,5
$$$$
2,4
$$$$
1,7
$$$$
2,5
$$

\begin{tabular}{|c|c|c|c|c|c|c|c|}
\hline Ap1 $(0-15 \mathrm{~cm})$ & 1,4 & 16,8 & 15,9 & 31,5 & 34,5 & 0,58 & 58,12 \\
\hline Ap2 $(15-34 \mathrm{~cm})$ & 6,3 & 29,8 & 19,4 & 22,0 & 22,5 & 0,98 & 70,11 \\
\hline $\mathrm{AB}(34-55 \mathrm{~cm})$ & 3,6 & 16,0 & 15,6 & 28,6 & 36,2 & 0,68 & 48,87 \\
\hline BA $(55-95 \mathrm{~cm})$ & 1,2 & 9,9 & 14,9 & 22,0 & 52,1 & 0,45 & 40,16 \\
\hline $\mathrm{Bw}_{1}(95-121 \mathrm{~cm})$ & 1,9 & 12,4 & 12,5 & 32,8 & 40,4 & 0,52 & 51,12 \\
\hline $\mathrm{Bw}_{2}(121-180 \mathrm{~cm}+)$ & 3,5 & 12,2 & 15,3 & 23,6 & 45,4 & 0,55 & 68,31 \\
\hline \multicolumn{8}{|c|}{ LAd6 - Latossolo Amarelo distrófico típico - mata nativa } \\
\hline A $\quad(0-26 \mathrm{~cm})$ & 6,1 & 38,7 & 14,0 & 21,8 & 19,5 & 1,00 & 81,48 \\
\hline $\mathrm{AB} \quad(26-46 \mathrm{~cm})$ & 3,5 & 19,3 & 12,3 & 24,8 & 40,0 & 0,65 & 64,57 \\
\hline BA $(46-74 \mathrm{~cm})$ & 2,2 & 10,3 & 9,4 & 17,8 & 60,3 & 0,43 & 50,92 \\
\hline $\mathrm{Bw}_{1}(74-118 \mathrm{~cm})$ & 0,5 & 7,6 & 11,6 & 17,9 & 62,4 & 0,39 & 27,37 \\
\hline $\mathrm{Bw}_{2}(118-195 \mathrm{~cm}+)$ & 0,6 & 5,0 & 9,1 & 17,0 & 68,3 & 0,31 & 43,19 \\
\hline
\end{tabular}

$\begin{array}{lll}25,1 & 20,1 & 26,0 \\ 20,5 & 14,6 & 19,8 \\ 10,1 & 11,7 & 27,6 \\ 13,6 & 19,4 & 24,1\end{array}$

LAd4 - Latossolo Amarelo distrófico húmico - pastagem braquiária

$\begin{array}{rrrrrrr}8,0 & 32,7 & 12,6 & 22,0 & 24,7 & 0,97 & 77,28 \\ 17,2 & 36,4 & 10,3 & 15,0 & 21,1 & 1,29 & 80,71 \\ 10,8 & 8,4 & 8,8 & 17,9 & 54,1 & 0,60 & 59,75 \\ 8,4 & 13,6 & 10,7 & 26,1 & 41,2 & 0,72 & 74,40 \\ 3,1 & 17,6 & 15,9 & 19,5 & 43,9 & 0,62 & 69,98\end{array}$

LAd5 - Latossolo Amarelo distrófico típico- café (cultivo não mecanizado)

DMP = diâmetro médio ponderado; IEA = índice de estabilidade de agregados. 
Enquanto o horizonte A da mata nativa (LAd6) exibiu $81,48 \%$ de IEA, o AB ficou com $64,57 \%$ e o BA com 50,92 \%, bem diferente da mata nativa da SBHRM, que apresentou um IEA próximo de $80 \%$ nesses horizontes, chegando, inclusive, a $84,85 \%$ no $\mathrm{AB}$. Possivelmente, a baixa agregação no LAd6 tem relação com o menor percentual de argila deste solo. Já o elevado IEA no horizonte A pode ser creditado ao papel cimentante da matéria orgânica, já que o C orgânico nesse horizonte foi igual ao encontrado na mata do rio dos Monos, cerca de $28,8 \mathrm{~g} \mathrm{dm}^{-3}$, contribuindo, também, para o aumento do DMP e dos agregados de maior diâmetro.

Também na SBHRAF, o solo sob cultura cafeeira foi o mais alterado pelo tipo de uso, exibindo menores valores de IEA, DMP e maior proporção de agregados de menor tamanho. Neste solo, o horizonte Ap1, embora tenha apresentado o maior teor de C orgânico (48,6 g $\mathrm{dm}^{-3}$ ), não conseguiu elevar os valores do IEA, DMP, tampouco o tamanho dos agregados, predominando os agregados de menor diâmetro. Isto pode estar relacionado com o baixo teor de argila e predominância da fração areia nesse horizonte, que foi o único a apresentar textura média. Nos dois ambientes de café (LAd3 e LAd5), foram notados os efeitos mais nocivos sob o solo, mas os dados indicam que o LAd3 apresentou as maiores alterações. Este fato pode ser evidenciado pela menor espessura do horizonte $\mathrm{A}(18 \mathrm{~cm})$ do solo em relação a todos os outros usos, sugerindo maiores taxas erosivas neste ambiente, e, em relação à estrutura do solo, as alterações são atestadas pelo menor IEA $(66,96 \%)$, menor DMP $(0,60 \mathrm{~mm})$ e menor percentual de agregados de maior diâmetro, uma vez que, nas classes de 4,76-2,00 mm e 2,00-1,00 mm, o LAd3 exibiu $26,6 \%$ no horizonte Ap. Comparativamente, o LAd5 apresentou maior espessura do horizonte A $(55 \mathrm{~cm})$ com maior subdivisão do horizonte (Ap1, Ap2 e AB), evidenciando um ambiente mais preservado em relação às perdas de solo. Em relação à estrutura, apesar de o horizonte Ap1 apresentar menor IEA, por razões explicadas anteriormente, o horizonte Ap2 apresentou maiores IEA $(70,11 \%)$, DMP $(0,98 \mathrm{~mm})$ e percentual de agregados de maior diâmetro (4,76-2,00 mm e 2,00$1,00 \mathrm{~mm}$ ) com $36,1 \%$. Isto demonstra que o manejo adotado no LAd5 - onde o solo não é submetido ao trânsito de máquinas agrícolas pesadas para os tratos culturais e colheita - foi mais eficiente na preservação do solo e da sua estrutura.

\section{Carbono orgânico}

Em termos gerais, todos os solos apresentaram teores elevados de C orgânico (Quadro 2) nos horizontes superficiais (> $\left.14 \mathrm{~g} \mathrm{dm}^{-3}\right)$. Uma explicação está relacionada com as características geomorfológicas da área. Os solos estão localizados na unidade denominada Planaltos dos Geraizinhos, com altimetria sempre superior a $800 \mathrm{~m}$, que favorece o estabelecimento de temperaturas mais baixas que acabam dificultando a decomposição da matéria orgânica, que se vai acumulando no perfil superior do solo (Bronick \& Lal,
2005). Outra explicação pode estar relacionada com a pobreza em nutrientes desses solos. A baixa disponibilidade de nutrientes dificulta a ação dos microrganismos decompositores (Killham, 1994; Resende et al., 2002), e o resultado disso é que a adição de material orgânico passa a ser maior do que a mineralização, com o conseqüente acúmulo de matéria orgânica.

Para uma mesma produção e deposição de biomassa vegetal ao solo, o teor de matéria orgânica (MO) pode variar de solo para solo, tendo em vista a influência de diversos fatores sobre a microbiota do solo e, conseqüentemente sobre a taxa de decomposição. Neste sentido, segundo Resende et al. (2002), a baixa temperatura, a deficiência em água, a falta de aeração (anaerobiose) e os baixos teores de nutrientes no solo inibem a ação dos microrganismos, podendo favorecer acúmulo de MO. Obviamente, se nenhum destes fatores for limitante à ação da microbiota do solo, a decomposição de MOS é acelerada.

Quanto aos efeitos provocados pelo tipo de uso, os solos localizados na SBHRM apresentaram alterações significativas relacionadas com a redução do teor de $\mathrm{C}$ orgânico no sentido mata-pastagem-café. Para o horizonte A, a mata nativa (LAd2) alcançou $28,80 \mathrm{~g}$ $\mathrm{dm}^{-3}$ de $\mathrm{C}$, contra $22,08 \mathrm{~g} \mathrm{dm}^{-3}$ de $\mathrm{C}$ da pastagem (LAd1) e 18,60 $\mathrm{g} \mathrm{dm}^{-3}$ de C do solo sob café (Ap do LAd3). Apesar das reduções verificadas, os teores são considerados médios, segundo CFSEMG (1999).

A ação das gramíneas, por meio do seu sistema radicular numeroso e bem distribuído pelos horizontes superficiais e sub-superficiais (Silva \& Mielniczuk, 1997; Cruz et al., 2003; Wohlenberg et al., 2004) pode ter contribuído para a manutenção de teores altos de $\mathrm{C}$ orgânico até à profundidade de $24 \mathrm{~cm}$.

Os teores de C orgânico do LAd3 foram os mais baixos dentre todos os solos estudados. O sistema de manejo adotado nesse cafezal, caracterizado pela menor densidade populacional de plantas de café e uso de máquinas para os tratos culturais e colheita, contribuiu para a fragmentação dos agregados que protegem a matéria orgânica, provocando a redução de seu teor (Bertol et al., 2004; Oliveira et al., 2004).

Na SBHRAF, o manejo adotado no solo sob café (LAd5), sistema semi-adensado, incrementou os teores de $\mathrm{C}$ orgânico nos horizontes superficiais e subsuperficiais até à profundidade de $55 \mathrm{~cm}$. O elevado teor de C orgânico encontrado no horizonte Ap1, cerca de $48,60 \mathrm{~g} \mathrm{dm}^{-3}$ de $\mathrm{C}$, é explicado pelo menor revolvimento (Centurion et al., 1985) e pela técnica de recobrimento do solo com "palha de café".

\section{CONCLUSÕES}

1. A magnitude dos efeitos causados pelos tipos de uso do solo nos atributos avaliados aumentou no sentido mata-pastagem-cafezal. 
2. O café sob sistema convencional apresentou os efeitos mais nocivos para a estrutura do solo, pela menor estabilidade de agregados, refletidos pelo menor diâmetro médio ponderado e pela maior quantidade de agregados de menor tamanho.

3. O café sob sistema semi-adensado incrementou os teores de C orgânico do solo, em relação aos encontrados nas áreas de mata, enquanto o sistema convencional resultou em forte redução nestes teores.

\section{LITERATURA CITADA}

BERTOL, I.; BEUTLER, J.F.; LEITE, D. \& BATISTELA, O. Propriedades físicas de um Cambissolo Húmico afetadas pelo tipo de manejo de solo. Sci. Agric., 58:555-560, 2001.

BERTOL, I.; ALBUQUERQUE, J.A.; LEITE, D.; AMARAL, A.J. \& ZOLDAN JUNIOR, W.A. Propriedades físicas do solo sob preparo convencional e semeadura direta em rotação e sucessão de culturas, comparadas às do campo nativo. R. Bras. Ci. Solo, 28:155-163, 2004.

BRASIL. Ministério das Minas e Energia. Secretaria Geral. Projeto RADAMBRASIL: Folha SD.24 Salvador: geologia, geomorfologia, pedologia, vegetação e uso potencial da terra. Rio de Janeiro, 1981. 624p.

BRONICK, C.J. \& LAL, R. Soil structure and management: A review. Geoderma, 124:3-22, 2005.

CENTURION, J.F.; DEMATTÊ, J L.I. \& FERNANDES, F.M. Efeitos de sistemas de preparo nas propriedades químicas de um solo sob Cerrado cultivado com soja. R. Bras. Ci. Solo, 9:267-270, 1985

COMISSÃO DE FERTILIDADE DO SOLO DO ESTADO DE MINAS GERAIS - CFSEMG. Recomendações para o uso de corretivos e fertilizantes em Minas Gerais. $5^{\circ}$ aproximação. Viçosa, MG, 1999. 359p.

CRUZ, A.C.R.; PAULETTO, E.A.; FLORES, C.A. \& SILVA, J.B. Atributos físicos e carbono orgânico de um Argissolo Vermelho sob sistemas de manejo. R. Bras. Ci. Solo, 27:1105-1112, 2003.
EMPRESA BRASILEIRA DE PESQUISA AGROPECUÁRIA EMBRAPA. Centro Nacional de Pesquisa de Solos. Manual de métodos de análise de solo. 2.ed. Rio de Janeiro, 1997. 212p.

GRIEVE, I.C.; DAVIDSON, D.A. \& BRUNEAU, P.M.C. Effects of liming on void space and aggregation in an upland grassland soil. Geoderma, 125:39-48, 2005.

JOHN, B.; YAMASHITA, T.; LUDWIG, B. \& FLESSA, H. Storage of organic carbon in aggregate and density fractions of silty soils under different types of land use. Geoderma, 128:63-79, 2005.

KILLHAM, K. Soil ecology. Cambrige, Cambrige University Press, 1994.

OLIVEIRA, G.C.; DIAS JUNIOR, M.S.; RESCK, D.V.S. \& CURI, N. Caracterização química e físico-hídrica de um Latossolo Vermelho após vinte anos de manejo e cultivo do solo. R. Bras. Ci. Solo, 28:327-336, 2004.

RESENDE, M.; CURI, N.; REZENDE, S.B. \& CORREAA, G.F. Pedologia: Base para distinção de ambientes. Viçosa, MG, NEPUT, 2002.

ROTH, C.H.; CASTRO FILHO, C. \& MEDEIROS, G.B. Análise de fatores físicos e químicos relacionados com agregação de um Latossolo Roxo distrófico. R. Bras. Ci. Solo, 15:241248, 1991.

SILVA, I.F. \& MIELNICZUK, J. Ação do sistema radicular de plantas na formação e estabilização de agregados do solo. R. Bras. Ci. Solo, 21:113-117, 1997.

WENDLING, B.; JUCKSCH, I.; MENDONÇA, E.S. \& NEVES, J.C.L. Carbono orgânico e estabilidade de agregados de um Latossolo Vermelho sob diferentes manejos. Pesq. Agropec. Bras., 40:487-494, 2005.

WOHLENBERG, E.V.; REICHERT, J.M.; REINERT, D.J. \& BLUME, E. Dinâmica da agregação de um solo francoarenoso em cinco sistemas de culturas em rotação e em sucessão. R. Bras. Ci. Solo, 28:891-900, 2004.

YOUKER, R.E. \& McGUINNESS, J.L. A short method of obtaining mean weight-diameter values of aggregate analyses of soils. Soil Sci., 83:291-294, 1957. 
